\title{
EXISTENCE OF EQUILIBRIUM STATES OF HOLLOW ELASTIC CYLINDERS SUBMERGED IN A FLUID
}

\author{
M.B.M. ELGINDI and D.H.Y. YEN \\ Department of Mathematics \\ Michigan State University \\ East Lansing, Michigan 48824
}

(Received November 14, 1990)

\begin{abstract}
This paper is concerned with the existence of equilibrium states of thin-walled elastic, cylindrical shell fully or partially submerged in a fluid. This problem obviously serves as a model for many problems with engineering importance. Previous studies on the deformation of the shell have assumed that the pressure due to the fluid is uniform. This paper takes into consideration the non-uniformity of the pressure by taking into account the effect of gravity. The presence of a pressure gradient brings additional parameters to the problem which in turn lead to the consideration of several boundary value problems.
\end{abstract}

KEY WORDS AND PHRASES. Existence of equilibrium states, hollow elastic cylinders, nonlinear eigenvalue problem, non-linear integro-differential equation, minimization of functional with several constraints, Browder Theorem, linearly independent operators.

1980 AMS SUBJECT CLASSIFICATION CODE. 49G99, 73H05, 73K15.

1. INTRODUCTION. We consider in this paper the existence of equilibrium states of hollow elastic cylinders submerged in a fluid. We treat the fluid pressure as non-uniform by taking gravity into account. To balance the bouyancy force in the fluid an external line load is applied to the bottom of the cylinder. By treating the problem as one independent of the variable along the axis of the cylinder we arrive at the same nonlinear ordinary differential equations governing an inextensible elastica as in Tadjbakhsh and Odeh [1] for a circular ring. Our work generalizes that in [1] where only uniform external pressures are considered.

In Section 2 we present the mathematical formulation. By seeking equilibrium states that are symmetric with the vertical $(y-)$ axis it suffices to consider one half of the perimeter $0<s<1$ where $s$ denotes the non-dimensional arc length. We present boundary value problems for a nonlinear integro-differential equation for the dependent function $\varphi=\varphi(s)$ which is the angle deviation from the circular state. An alternative formulation in terms of $w=\varphi_{s}$ is also presented.

The mathematical problems formulated here involve two non-dimensional pressure parameters $\lambda$ and $\tau$, where $\lambda$ corresponds to the uniform part of the pressure while $\tau$ corresponds to the pressure gradient. When $\tau=0$, the problems reduce to those considered in [1]. When $\tau \neq 0$, bouyancy force arises which depends on $\tau$ as well as on the equilibrium configuration and is balanced by the applied force $2 \Gamma$ at the bottom of the hollow cylinder. The concentrated force $2 \Gamma$ 
gives rise to a shear force $\Gamma$ at $s=1^{-}$because of symmetry. We assume that $\Gamma$ is given in Theorem 3.1 in Section 3.

The function $w=\varphi_{s}$ is a measure of the deviation of the curvature from that of the circular state and the square of the $L_{2}$-norm of $w$, denoted by $K$, is a measure of the bending energy. It was observed in [1] that when $\tau=0$ the circular state is always an equilibrium solution for which $K=0$ regardless of $\lambda$. Non-circular equilibrium states with increasing degrees of symmetry, however, bifurcate from the circular state at a set of buckling pressures. One main result in [1] is that given $K$, there exist an infinite set of such non-circular equilibrium states. Our Theorem 3.1 in Section 3, in the $w$-formulation and with given $\Gamma$ and $K$, generalizes the above result in [1].

As in [1] our main mathematical tools come from the variational methods for nonlinear elliptic eigenvalue problems. In Section 3 we state Lemma 3.2 which is used in the proofs of the theorems in both Sections 3 and 4. This lemma is a direct generalization of the results of Browder [2] to the case where several constraints are involved.

In Section 4 we consider cases where $K$ is not given. For such cases it is more convenient to work with the $\varphi$-formulation of Section 2. In Theorem 4.1 we establish the existence of solution with given $\Gamma$ and $\tau$, provided they satisfy the inequality $\Gamma<\tau / 2 \pi$. In Theorem 4.2 we establish the existence of solution when $\lambda$ and $\tau$ are given. Finally in Theorem 4.3 we consider the case of partially submerged cylinders and establish the existence of solution with given $\tau$.

Some discussions are given in Section 5.

2. THE MATHEMATICAL FORMULATION.

We consider a typical cross section of a hollow cylinder as shown in Figure 1. Let $\left(x^{\prime}, y^{\prime}\right)$ be the Cartesian coordinates with origin at $\mathrm{O}, s^{\prime}$ the arc length measured from $\mathrm{O}$, and $\theta$ the local angle. The hydrostatic pressure $p\left(s^{\prime}\right)$ at a point $\left(x^{\prime}, y^{\prime}\right)$ (per unit length along the cylinder) is

$$
p\left(s^{\prime}\right)=p_{o}+\rho g y^{\prime}
$$

where $p_{o}$ is the external pressure at $s^{\prime}=0, \rho$ the fluid density, and $g$ the gravitational acceleration. Balancing moments on an element of length $d s^{\prime}$ as shown in Figure 2 yields

$$
\frac{d m}{d s^{\prime}}=\left(-h^{\prime}+\int_{0}^{s^{\prime}} p(t) \sin \theta(t) d t\right) \sin \theta+\left(\int_{0}^{s^{\prime}} p(t) \cos \theta(t) d t\right) \cos \theta,
$$

where $h^{\prime}$ is the horizontal component of the internal force at $s^{\prime}=0$.

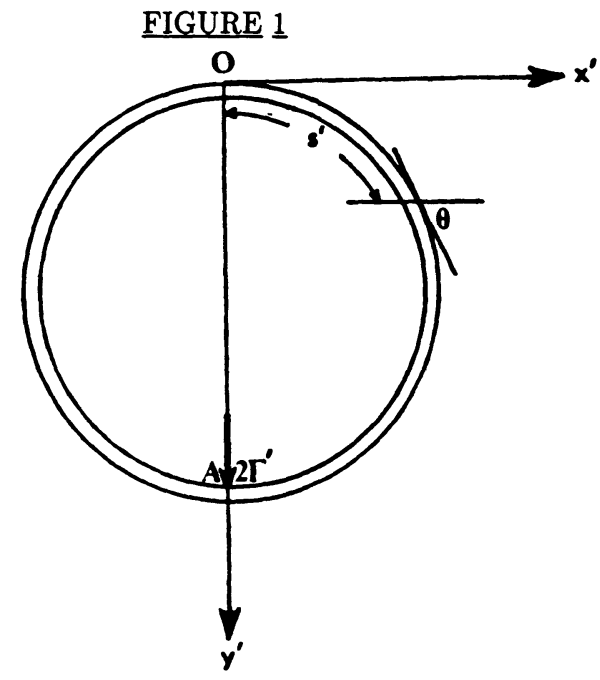

FIGURE 2

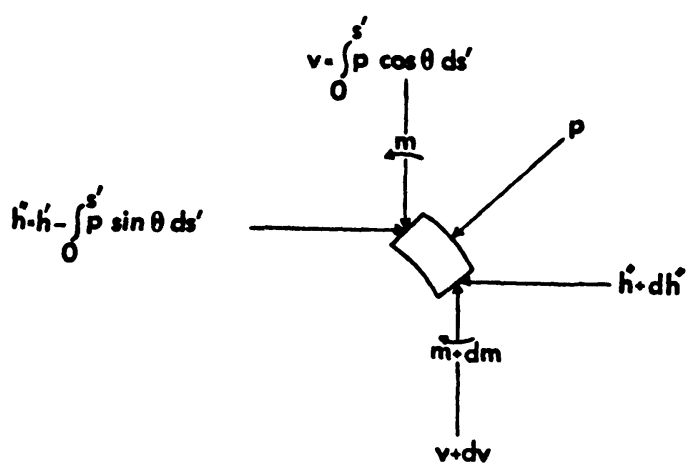


The local bending moment $m$ and the Cartesian coordinates $x^{\prime}$ and $y^{\prime}$ are related to $\theta$ and $s^{\prime}$ by

$$
m=E I \frac{d \theta}{d s^{\prime \prime}}, \quad \frac{d x^{\prime}}{d s^{\prime}}=\cos \theta, \quad \frac{d y^{\prime}}{d s^{\prime}}=\sin \theta,
$$

where $E I$ is the flexural rigidity of the cylinder.

The following non-dimensional quantities are introduced:

$$
s=\frac{s^{\prime}}{L}, \quad x=\frac{x^{\prime}}{L}, \quad y=\frac{y^{\prime}}{L}, \quad \lambda=\frac{p_{o} L^{3}}{E I}, \quad \tau=\frac{\rho g L^{4}}{E I}, \quad h=\frac{h^{\prime} L^{2}}{E I}, \quad \Gamma=\frac{\Gamma^{\prime} L^{2}}{E I}
$$

where $2 L$ is the perimeter of the cylinder and $2 \Gamma^{\prime}$ is the external vertical force applied at the point $A$. Equations (2.1), (2.2) and (2.3) now lead to

$$
\begin{gathered}
\theta_{s s}=-h \sin \theta+\lambda(x \cos \theta+y \sin \theta)+\tau\left(u \cos \theta+\frac{1}{2} y^{2} \sin \theta\right), \\
x_{s}=\cos \theta, \\
y_{s}=\sin \theta,
\end{gathered}
$$

and

$$
u=\int_{0}^{s} y(t) \cos \theta(t) d t \quad\left(u_{s}=y \cos \theta\right) .
$$

By integrating (2.5b) and (2.5c) we can show that

$$
x \cos \theta+y \sin \theta=\int_{0}^{s} \cos (\theta(s)-\theta(t)) d t .
$$

Making the change of variables

$$
\varphi=\theta-\pi s,
$$

where $\varphi$ measures the deviation of $\theta$ from the circular configuration, we obtain from (2.5a) the following equation for $\varphi=\varphi(s)$

$$
\begin{aligned}
\varphi_{s s} & =-h \sin (\varphi+\pi s)+\lambda \int_{0}^{s} \cos (\varphi(s)-\varphi(t)+\pi s-\pi t) d t \\
& +\tau\left[u \cos (\varphi+\pi s)+\frac{1}{2} y^{2} \sin (\varphi+\pi s)\right] .
\end{aligned}
$$

We seek equilibrium states that are symmetric about the $y$-axis and confine ourselves to the interval $0<s<1$. Since $\theta(0)=0$ and $\theta(1)=\pi$ we have

Also by $x(1)=0$ we have by $(2.5 \mathrm{~b})$

$$
\varphi(0)=\varphi(1)=0 .
$$

$$
x(1)=\int_{0}^{1} \cos (\varphi+\pi t) d t=0 .
$$

The shear force has a jump of $2 \Gamma$ across $s=1$. Hence

$$
\varphi_{s s}\left(1^{-}\right)=\Gamma \text {. }
$$


The functions $y$ and $u$ in (2.8) are now integrals of $\varphi$ given by

$$
\begin{gathered}
y(s)=\int_{0}^{s} \sin (\varphi(t)+\pi t) d t, \\
u(s)=\int_{0}^{s} y(t) \cos (\varphi(t)+\pi t) d t .
\end{gathered}
$$

Thus we may regard (2.8) as an integro-differential equation for $\varphi$. The boundary value problem described by (2.8) through (2.10) will be considered in Section 4.

In the remainder of this section we shall present an alternative formulation of the problem, which is then studied in Section 3. Differentiating (2.8) with respect to $s$ one more time and introducing the new variable $w$,

$$
w=\varphi_{s}
$$

the following integro-differential equation for $w$ results

where

$$
w_{s s}+\nu w=\delta-f(w)-\tau \int_{0}^{s} \sin \left(\pi t+\int_{0}^{t} w(\xi) d \xi\right) d t
$$

$$
\nu=\frac{3}{2} \pi^{2}-c, \quad \delta=\lambda+c \pi-\frac{\pi^{3}}{2}, \quad f(w)=\frac{w^{3}}{2}+\frac{3 \pi w^{2}}{2} .
$$

The constant $c$ above arises as an integration constant and is related to $h$ by

$$
c=\frac{1}{2} \theta_{s}^{2}(0)-h=\frac{1}{2}\left[\varphi_{s}(0)+\pi\right]^{2}-h .
$$

For symmetric equilibrium states about the $\mathrm{y}$-axis we again consider (2.12) in $0<s<1$ with the boundary conditions

$$
\begin{gathered}
w_{s}(0)=0, \quad w_{s}(1)=\Gamma \\
\int_{0}^{1} w d s=0 \\
\int_{0}^{1} \cos \left(\pi s+\int_{0}^{s} w(\xi) d \xi\right) d s=0 .
\end{gathered}
$$

We shall also assume that the $L_{2}$-norm of $w$ is fixed. The boundary value problem for $w$ described by (2.12) through (2.15) will be studied in the next section.

We remark that with $\tau=0$ and $\Gamma=0$, the above formulations correspond to those in [1], though a different length normalization than that in [1] has been used here.

\section{AN EXISTENCE THEOREM FOR THE $w$-PROBLEM WITH GIVEN TOTAL BENDING} ENERGY.

In this section we shall consider the existence of solutions of the $w$-problem described above with

$$
\int_{0}^{1} w^{2} d s=K
$$

where $K$ is given. Since the function $w$ introduced in (2.11) is a measure of the departure of the curvature from the circular state, $K$ in (3.1) is a measure of the bending energy. When $\tau=0$, it was established in [1] that for $K>0$, non-circular equilibrium states with increasing degrees of 
symmetry exist and bifurcate from the circular state at a set of buckling pressures. The following theorem generalizes the above result in [1].

THEOREM 3.1. For any given $\Gamma>0$ and $K>0$, there exist $\nu, \delta, \tau$ in $\mathbb{R}$ and $w$ in the Sobolev space $W^{1,2}((0,1))$ such that $(w, \nu, \delta, \tau)$ is a weak solution of $(2.12)$ satisfying $(2.15)$ and (3.1).

To prove Theorem 3.1 as well as Theorem 4.1 through Theorem 4.3 in Section 4 we state below a lemma that will be our main tool.

LEMMA 3.2. Let $H$ be a Hilbert space and $\Phi$ a semi-convex real functional on $H \times H$. Let $E(u)=\Phi(u, u)$ be differentiable for $u$ in $H$. Let $g_{i}(u), i=1,2, \cdots, N$ be weakly continuous and differentiable on $H$. Given the constants $c_{i}$ in $\mathbf{R}$ we assume that the set

$$
C \equiv\left\{u \in H: g_{i}(u)=c_{i}, \quad i=1,2, \cdots, N\right\}
$$

is either (i) bounded in $H$ or (ii) $E(u) \rightarrow+\infty$ as $\|u\| \rightarrow \infty$ on $C$ ( $\|\cdot\|$ denotes the Hilbert space norm). Then there exist constants $\lambda_{i}, i=1,2, \cdots, N$ in $\mathbb{R}$ and some $w$ in $C$ such that

$$
E^{\prime}(w)-\sum_{i=1}^{N} \lambda_{i} g_{i}^{\prime}(w)=0 \quad \text { on } H
$$

provided that the operators, $g_{i}^{\prime}(w), i=1,2, \cdots, N$, are linearly independent.

REMARK 3.3. Lemma 3.2 is a generalization of Theorem 5 in [2]. The theorems in [2] are for general reflexive Banach spaces which we specialize here to Sobolev spaces that are Hilbert spaces. Lemma 3.2 generalizes the result in [2] to the case where $N$ constraints are involved. In the applications below $N \leq 3$.

REMARK 3.4. The derivatives in (3.3) are Frechét derivatives and in the applications below they correspond to linear integral operators on $H$.

REMARK 3.5. The assumption made on the set $C$ in Lemma 3.2 above guarantees the existence of a minimizer $w$ in the interior of a large closed ball with radius $R$, $B_{R} \equiv\{v \in H:\|v\| \leq R\}$, for which (3.3) holds. For details see [2].

PROOF OF THEOREM 3.1. To apply Lemma 3.2 we let

and define the functionals

$$
H \equiv W^{1,2}((0,1))
$$

$$
\begin{gathered}
\Phi(v, w) \equiv \frac{1}{2} \int_{0}^{1} v_{s}^{2} d s-\int_{0}^{1}\left(\frac{1}{8} w^{4}+\frac{1}{2} \pi w^{3}\right) d s-\Gamma v(1) \\
g_{1}(w)=\int_{0}^{1} w d s \\
g_{2}(w)=\int_{0}^{1} w^{2} d s \\
g_{3}(w) \equiv \int_{0}^{1} \cos \left(\pi s+\int_{0}^{s} w(\xi) d \xi\right) d s
\end{gathered}
$$

By arguments similar to those in [1] we can show that the functionals above have the following properties:

(i) $\Phi$ is differentiable and semi-convex on $H \times H$;

(ii) Each of $g_{i}, i=1,2,3$, is differentiable and weakly continuous on $H$; and 
(iii) $\quad E(w) \equiv \Phi(w, w) \rightarrow \infty$ as $\|w\| \rightarrow \infty$ on the set

$$
C \equiv\left\{w \in H: g_{i}(w)=c_{i}, \quad i=1,2,3\right\}
$$

where $c_{1}=0, c_{2}=K, c_{3}=0$, for any $K>0$. By Lemma 3.2 there exist some $w$ in $C$ and $\lambda_{i}$, $i=1,2,3$ in $\mathbf{R}$ such that $(3.3)$ holds on $H$ provided that the operators $\left\{g_{i}^{\prime}(w)\right\}, i=1,2,3$, are linearly independent. Thus, by (3.3) we have

for every $v$ in $H$. We can show that

$$
E^{\prime}(w) v-\sum_{i=1}^{N} \lambda_{i} g_{i}^{\prime}(w) v=0
$$

$$
\begin{gathered}
g_{1}^{\prime}(w) v=\int_{0}^{1} v d s \\
g_{2}^{\prime}(w) v=2 \int_{0}^{1} w v d s
\end{gathered}
$$

and

$$
\begin{aligned}
g_{3}^{\prime}(w) v & =-\int_{0}^{1} \sin \left(\pi t+\int_{0}^{t} w(\xi) d \xi\right) d t \int_{0}^{1} v d s \\
& +\int_{0}^{1}\left[\int_{0}^{s} \sin \left(\pi t+\int_{0}^{t} w(\xi) d \xi\right) d t\right] v(s) d s
\end{aligned}
$$

Substituting (3.11a) through (3.11c) into (3.10) yields

where

$$
\begin{aligned}
E^{\prime}(w) v & +\nu \int_{0}^{1} w(s) v(s) d s+\tau \int_{0}^{1}\left[\int_{0}^{s} \sin \left(\pi t+\int_{0}^{t} w(\xi) d \xi\right) d t\right] v(s) d s \\
& =\delta \int_{0}^{1} v(s) d s
\end{aligned}
$$

Computing $E^{\prime}(w) v$ we can show that (3.12) becomes the weak form of (2.12), with (2.15a) satisfied as natural boundary conditions. The constraints $(2.15 \mathrm{~b}),(2.15 \mathrm{c})$ and $(3.1)$ are all satisfied by the definitions of $g_{i}(w)=c_{i}, i=1,2,3$.

It remains now to prove that the operators $g_{i}^{\prime}(w), i=1,2,3$ defined by $(3.11 \mathrm{a})-(3.11 \mathrm{c})$ are linearly independent for any $w$ in the set $C$ defined by (3.9). The proof is by contradiction. Thus, we assume that for some $w \in C$ the relation

$$
\mu_{i} g_{1}^{\prime}(w) v+\mu_{2} g_{2}^{\prime}(w) v+\mu_{3} g_{3}^{\prime}(w) v=0
$$

holds for every $v \in H$ and some $\mu_{1}, \mu_{2}, \mu_{3} \in \mathbf{R}$ which are not all zeros. Letting $v$ in (3.14) be 1 and $w$ respectively we get

$$
\begin{gathered}
\mu_{1}+\mu_{3} g_{3}^{\prime}(w) 1=0 \\
2 \mu_{2} K+\mu_{3} g_{3}^{\prime}(w) w=0 .
\end{gathered}
$$


From (3.15) and (3.16) it follows that $\mu_{3} \neq 0$ and hence (3.14) implies

$$
\int_{0}^{1} v(s)\left[c_{o}+c_{1} w(s)+\int_{0}^{s} \sin \left(\pi t+\int_{0}^{t} w(\xi) d \xi\right) d t\right] d s=0
$$

for each $v \in H$, where

$$
\begin{aligned}
& c_{o}=\frac{\mu_{1}}{\mu_{3}}-\int_{0}^{1} \sin \left(\pi t+\int_{0}^{t} w(\xi) d \xi\right) d t \\
& c_{1}=\frac{2 \mu_{2}}{\mu_{3}} .
\end{aligned}
$$

But, since $H$ is dense in $L_{2}((0,1)),(3.17)$ holds for every $v \in L_{2}((0,1))$, and hence implies

$$
c_{0}+c_{1} w(s)+\int_{0}^{s} \sin \left(\pi t+\int_{0}^{t}(w(\xi) d \xi)\right) d t=0 .
$$

Differentiating (3.18) with respect to $s$ yields

$$
c_{1} w_{s}=-\sin \left(\pi s+\int_{0}^{s} w(\xi) d \xi\right)
$$

We shall consider two cases:

CASE 1. $c_{1}=0$ (i.e., $\mu_{2}=0$ ). In this case (3.19) implies

$$
\pi s+\int_{0}^{s} w(\xi) d \xi=\text { constant }
$$

which implies that $w=-\pi \notin C$, and this is a contradiction.

CASE 2. $c_{1} \neq 0$. In this case (3.19) gives

or, in terms of the local angle $\theta$,

$$
w_{s}=-\frac{1}{c_{1}} \sin \left(\pi s+\int_{0}^{s} w(\xi) d \xi\right)
$$

$$
\theta_{s s}=-\frac{1}{c_{1}} \sin \theta \text {. }
$$

Taking $v=\cos \left(\pi s+\int_{0}^{s} w(\xi) d \xi\right)$ in (3.14) we get (since $w \in C, \int_{0}^{1} v d s=0$ and $\mu_{3} \neq 0$ )

which in terms of $\theta$ reads

$$
g_{3}^{\prime}(w) v=0
$$

$$
\int_{0}^{1} y \cos \theta d s=0
$$

Now (3.21) implies that $\theta$ cannot increase from 0 to $\pi$ on $[0,1]$ monotonically, i.e., there must be some $s^{*} \in[0,1]$ such that $\theta_{s}\left(s^{*}\right)=0$. Let $\theta^{*}$ be $\theta\left(s^{*}\right)$. Integrating (3.20) with respect to $\theta$ and using the condition $\theta_{s}\left(s^{*}\right)=0$ we get

$$
\frac{\theta_{s}^{2}}{2}=\frac{1}{c_{1}}\left(\cos \theta-\cos \theta^{*}\right) .
$$

Since $\theta(0)=0$ and $\theta(1)=\pi$, it follows from (3.22) that $\theta^{*}=n \pi$ for some integer $n$. However, the 
initial value problem

$$
\begin{aligned}
& \theta_{s s}=-\frac{1}{c_{1}} \sin \theta, \\
& \theta\left(s^{*}\right)=n \pi, \\
& \theta_{s}\left(s^{*}\right)=0,
\end{aligned}
$$

has the unique solution, $\theta=n \pi$. Since $\theta=n \pi$ cannot satisfy both the conditions $\theta(0)=0$ and $\theta(1)=\pi$, we have a contradiction.

REMARK. Theorem 3.1 above establishes the existence of non-circular equilibrium states that are symmetric about the $y$-axis for given bouyancy force $2 \Gamma$ and bending energy $K$. As suggested by [1] we expect that there are many such solutions which for small $\Gamma$ are perturbations of those obtained in [1] possessing high degrees of symmetry, though such latter symmetry is lost in the present solutions with $\tau \neq 0$.

\section{EXISTENCE OF SOLUTIONS FOR GIVEN PRESSURE GRADIENTS.}

In this section we shall establish several existence theorems with $\tau$ being given but with $K$ unspecified. In such cases it is more convenient to work with the $\varphi$-formulation described in Section 2.

In the first theorem below both $\tau$ and $\Gamma$ are given. It turns out that they must satisfy the inequality $\Gamma<\tau / 2 \pi$ which has a simple geometric interpretation to be made below.

THEOREM 4.1. For given values of $\tau>0$ and $\Gamma>0$ such that $\Gamma<\tau / 2 \pi$, there exists a weak solution $(\varphi, h, \lambda)$ of the boundary value problem described by $(2.8)$ through $(2.10)$.

PROOF. To prove this theorem using Lemma 3.2 we let

$$
H \equiv W_{0}^{1,2}((0,1))
$$

and define the functionals

$$
\begin{gathered}
\Phi(\psi, \varphi) \equiv \frac{1}{2} \int_{0}^{1} \psi_{s}^{2} d s+\tau \int_{0}^{1}\left[u(\varphi) \sin (\varphi+\pi t)-\frac{1}{2} y^{2}(\varphi) \cos (\varphi+\pi t)\right] d t \\
g_{1}(\varphi) \equiv \int_{0}^{1} \cos (\varphi+\pi t) d t \\
g_{2}(\varphi) \equiv \int_{0}^{1} y(\varphi) \cos (\varphi+\pi t) d t
\end{gathered}
$$

We can show that these functionals have the following properties:

(i) $\Phi$ is differentiable and semi-convex on $H \times H$;

(ii) Each of $g_{1}, i=1,2$, is differentiable and weakly continuous on $H$; and

(iii) $E(\varphi) \equiv \Phi(\varphi, \varphi) \rightarrow \infty$ as $\|\varphi\| \rightarrow \infty$ on the set

where $c_{1}=0, c_{2}=-\Gamma / \tau$.

$$
C \equiv\left\{\varphi \in H: g_{i}(\varphi)=c_{i}, i=1,2\right\}
$$

By Lemma 3.2 there exists some element $\varphi$ in $C$ and constants $\lambda_{1}$ and $\lambda_{2}$ in $\mathbb{R}$ such that

$$
E^{\prime}(\varphi)-\sum_{i=1}^{2} \lambda_{i} g_{i}^{\prime}(\varphi)=0
$$


provided that the set $C$ is nonempty and that $g_{1}^{\prime}(\varphi)$ and $g_{2}^{\prime}(\varphi)$ are linearly independent. Suppose that this is indeed the case. By the relations

and

$$
\frac{d}{d \theta}\left(u \sin \theta-\frac{1}{2} y^{2} \cos \theta\right)=u \cos \theta+\frac{1}{2} y^{2} \sin \theta,
$$

$$
\begin{aligned}
g_{2}^{\prime}(\varphi) \psi & =\int_{0}^{1} \frac{d}{d \varphi}[y(\varphi) \cos (\varphi+\pi t)] \psi(t) d t \\
& =\int_{0}^{1}\left[\int_{0}^{s} \cos (\varphi(s)-\varphi(t)+\pi s-\pi t) d t\right] \psi(s) d s,
\end{aligned}
$$

it easily follows that with $h=-\lambda_{1}$, and $\lambda=-\lambda_{2},(\varphi, h, \lambda)$ is a weak solution of the boundary value problem described by (2.8) through (2.10).

We now show that the set $C$ is nonempty for $\Gamma<\tau / 2 \pi$. To this end we note that for any given $\tau>0$, the total bouyancy force is $2 \Gamma=-\tau u(2)$ where $-u(2)$ is the total area enclosed in the hollow cylinder. By symmetry $u(2)=2 u(1)$ and since $u(1)=g_{2}(\varphi)$ we have $\Gamma=\tau g_{2}(\varphi)$. Clearly $-u(1)=-g_{2}(\varphi)$ has its maximum value $1 / 2 \pi$ when $\varphi(s)$ corresponds to a circle, i.e., $\varphi(s) \equiv 0$. Thus $\Gamma<\tau / 2 \pi$. By deforming the circle continuously through a family of ellipses symmetric about the $y$-axis $-g_{2}(\varphi)$ takes all the values in $(0,1 / 2 \pi)$ while $g_{1}(\varphi)=0$. Thus for $\Gamma<\tau / 2 \pi$ there exists , some $\varphi(s)$ for which $g_{1}(\varphi)=0$ and $g_{2}(\varphi)=-\Gamma / \tau$ and the set $C$ in (4.3) is nonempty.

For $0<\Gamma / \tau<1 / 2 \pi$ we now show that the operators $g_{1}^{\prime}(\varphi)$ and $g_{2}^{\prime}(\varphi)$ are linearly independent for any $\varphi$ in the set $C$ defined by (4.3). The proof is by contradiction. Suppose that

$$
g_{1}^{\prime}(\varphi) \psi=\delta g_{1}^{\prime}(\varphi) \psi
$$

holds for every $\psi \in H$ and some $\delta \neq 0$ in $\mathbf{R}$. Then (4.7) holds for each $\psi \in L_{2}((0,1))$ and this implies that

$$
\sin (\varphi+\pi s)=\delta \int_{0}^{s} \cos (\varphi(s)-\varphi(t)+\pi s-\pi t) d t
$$

or, in terms of $\theta$, that

or

$$
\sin \theta=\delta \int_{0}^{s} \cos (\theta(s)-\theta(t)) d t
$$

$$
\sin \theta=\delta(x \cos \theta+y \sin \theta) \text {. }
$$

However, we have

$$
\frac{d}{d \theta}(x \sin \theta-y \cos \theta)=x \cos \theta+y \sin \theta
$$

which allows us to integrate (4.8) with respect to $\theta$ to obtain

$$
-\cos \theta=\delta(x \sin \theta-y \cos \theta)+c,
$$

where $c$ is an integration constant. Differentiating (4.8) with respect to $s$ leads to

$$
\theta_{s} \cos \theta=\delta-\delta \theta_{s}(x \sin \theta-y \cos \theta) .
$$


From (4.9) and (4.10) it follows that

or (since $\delta \neq 0$ implies $c \neq 0$ )

$$
c \theta_{s}=-\delta
$$

$$
\theta_{s}=\frac{-\delta}{c}
$$

Since $\theta(0)=0$ and $\theta(1)=\pi,(4.11)$ implies that $\theta=\pi s$. Thus $\varphi=0 \notin C$, and this is a contradiction.

Thus the proof of Theorem 4.1 is completed.

REMARK. For $\Gamma=\tau / 2 \pi$ the above argument shows that the set $C$ consists only of the function $\varphi(s) \equiv 0$ which corresponds to the circular state. It also implies that $g_{1}^{\prime}(\varphi)$ and $g_{2}^{\prime}(\varphi)$ with $\varphi(s) \equiv 0$ are not linearly independent.

In the theorem below we assume that both the pressure parameters $\lambda$ and $\tau$ are given. The bouyancy force $2 \Gamma$ is then to be determined from

as part of the solution.

$$
\Gamma=-\tau \int_{0}^{1} y(t) \cos (\varphi(t)+\pi t) d t
$$

THEOREM 4.2. For given values of $\lambda>0$ and $\tau>0$ there exists a weak solution $(\varphi, h)$ of the boundary value problem described by (2.8), (2.9a), (2.9b) and (2.10).

PROOF. We let

$$
H \equiv W_{0}^{1,2}((0,1))
$$

and define the functionals

$$
\begin{aligned}
& \Phi(\psi, \phi) \equiv \frac{1}{2} \int_{0}^{1} \psi_{s}^{2} d s+\tau \int_{0}^{1}\left[u(\varphi) \sin (\varphi+\pi t)-\frac{1}{2} y^{2}(\varphi) \cos (\varphi+\pi t)\right] d t \\
&+\lambda \int_{0}^{1} \cos (\varphi(s)-\varphi(t)+\pi s-\pi t) d t \\
& g_{1}(\varphi) \equiv \int_{0}^{1} \cos (\varphi+\pi t) d t
\end{aligned}
$$

We can again show that these functionals have the following properties:

(i) $\Phi$ is differentiable and semi-convex on $H \times H$;

(ii) $g_{1}$ is differentiable and weakly continuous on $H$; and

(iii) $E(\varphi) \equiv \Phi(\varphi, \varphi) \rightarrow \infty$ as $\|\varphi\| \rightarrow \infty$ on the set

$$
C=\left\{\varphi \in H: g_{1}(\varphi)=0\right\}
$$

By Lemma 3.2 there exists some element $\varphi$ in $C$ and $\lambda_{1}$ in $\mathbf{R}$ such that

$$
E^{\prime}(\varphi)-\lambda_{1} g_{1}^{\prime}(\varphi)=0
$$

since it can be checked that $g_{1}^{\prime}(\varphi)$ cannot be the zero operator. Operating (4.15) on $\psi$ in $H$ with $h=-\lambda_{1}$ establishes $(\varphi, h)$ as a weak solution of the boundary value problem described by $(2.8)$, $(2.9 \mathrm{a}),(2.9 \mathrm{~b})$ and $(2.10)$.

We conclude this section by considering a partially submerged cylinder. Again we consider 
equilibrium states that are symmetric about the $y$-axis and restrict $s$ to be in $0<s<1$.

Let $r^{*}, 0<r^{*},<1$, represent the normalized arc length of the non-wetted part of the cylinder. We modify $(2.5 \mathrm{a})$ to

where

$$
\theta_{s s}=-h \sin \theta+\tau f(s)\left[u \cos \theta+\frac{1}{2}\left(y-y^{*}\right)^{2} \sin \theta-y^{*}\left(x-x^{*}\right) \cos \theta\right],
$$

$$
\begin{gathered}
f(s)= \begin{cases}0 & 0 \leq s \leq r^{*} \\
1 & r^{*}<s \leq 1,\end{cases} \\
x=\int_{0}^{s} \cos \theta(t) d t, \\
y=\int_{0}^{s} \sin \theta(t) d t, \\
u=\int_{r^{*}}^{s} y(t) \cos \theta(t) d t, \\
x^{*}=x\left(r^{*}\right), \quad y^{*}=y\left(r^{*}\right) .
\end{gathered}
$$

For given $\tau \geq 0$ we seek a weak solution $(\theta, h)$ of the above equations subject to

$$
\begin{gathered}
\theta(0)=0, \quad \theta(1)=\pi, \\
\int_{0}^{1} \cos \theta d s=0 .
\end{gathered}
$$

Alternatively, making the change of variables $\varphi=\theta-\pi s$ as in (2.7) enables us to work in the Hilbert space

$$
H=W_{0}^{1,2}((0,1))
$$

with the functionals similar to those in (4.15) defined by

$$
\begin{gathered}
\Phi(\psi, \varphi)=\frac{1}{2} \int_{0}^{1} \varphi_{s}^{2} d s+\tau \int_{0}^{1} f(s) G(\varphi) d s \\
g_{1}(\varphi)=\int_{0}^{1} \cos (\varphi+\pi t) d t
\end{gathered}
$$

where

$$
G(\varphi) \equiv \sin (\varphi+\pi s)-\frac{1}{2}\left(y-y^{*}\right)^{2} \cos (\varphi+\pi s)-y^{*}\left(x-x^{*}\right) \sin (\varphi+\pi s) .
$$

It can be shown here that $\Phi, E$ and $g_{1}$ all have the desired properties so that Lemma 3.2 may be applied. We summarize the above result in:

THEOREM 4.3. For each $\tau \geq 0$, there exists a weak solution $(\theta, h)$ of the boundary value problem described by (4.16) and (4.17) above.

5. DISCUSSIONS.

We have been concerned with the existence of equilibrium states of hollow elastic cylinders submerged in a fluid. By considering "planar" deformations of the hollow cylinders we used the same inextensible nonlinear elastica model as in [1] for elastic rings. 
In [1] Tadjbakhsh and Odeh established the existence of equilibrium states of elastic rings under uniform external pressures. Earlier works on elastic rings under uniform pressures were reviewed in [1]. A related note on this subject was given by S. Antmann [3]. Several later works by Antmann [4-6] also dealt with elastic rings under uniform external pressures, but the mathematical equations used went beyond the inextensible elastica model. In the work here we have treated the fluid pressure as nonuniform by taking gravity into account. Our results are thus applicable for elastic rings under nonuniform pressures.

By considering the pressure gradient in the fluid, the problem is complicated due to the fact that bouyancy force now arises. This bouyancy force, which also depends on the equilibrium states, is balanced by a concentrated force applied at the bottom of the cylinder and appears as an unknown boundary condition. Due to the additional parameters several different boundary value problems were formulated. The existence of solutions for equilibrium states in such problems were established in Theorems 3.1, 4.1 and 4.2 above. In Theorem 4.3 we have also considered the case of a partially submerged cylinder.

As in [1] we followed the mathematical tools provided by Browder in [2]. To facilitate our proofs we presented a generalization of Browder's results in Lemma 3.2 for semi-convex functionals in Hilbert spaces subject to several constraints.

One of the main concerns in [1] is the study of the existence of non-circular solutions that bifurcate from the trivial circular state at and beyond the buckling uniform pressures. The inclusion of a pressure gradient "perturbs" such bifurcations, much in the same way as an eccentric force does to the Euler's column buckling. We have examined in [7] details of this perturbed bifurcation picture near the first buckling pressure by singular perturbation techniques. For given pressure parameters we have also treated in [7] the nonlinear boundary value problems numerically by the shooting method, both away from and near the buckling pressures. As we have expected, symmetries of the solutions, except about the y-axis, are lost in the presence of the pressure gradient.

\section{REFERENCES}

1. TADJBAKHSK, I. and ODEH, F., Equilibrium states of elastic rings. J. Math. Anal. Appl. $\underline{18}$ (1967), 59-74.

2. BROWDER, F., Variational methods for non-linear elliptic eigenvalue problems. Bull. Amer. Math. Soc. 71 (1965), 176-183.

3. ANTMANN, S.S., A note on a paper of Tadjbakhsh. J. Math. Anal. 21 (1968), 132-135.

4. ANTMANN, S.S., General solutions for plane extensible elasticae having nonlinear stress-strain laws. Quart. Appl. Math. 26 (1968), 35-47.

5. ANTMANN, S.S., The shape of buckled nonlinearly elastic rings. Z.A.M.P. 21 (1970), 422438.

6. ANTMANN, S.S., Existence of solutions of equilibrium equations for nonlinearly elastic rings and arches. Indiana University Math. J. 20 No. 3 (1970), 281-302.

7. ELGINDI, M.B.M., "Deformation and load-carrying capacity of hollow elastic cylinders submerged in a fluid, Ph.D. Thesis, Department of Mathematics, Michigan State University, Sept. 1987. 


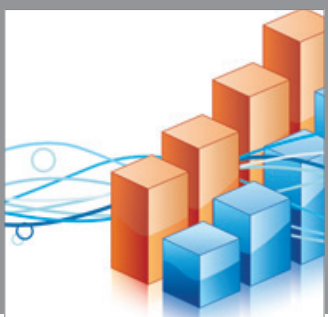

Advances in

Operations Research

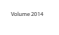

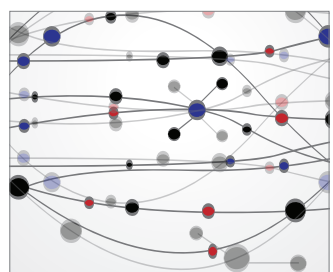

\section{The Scientific} World Journal
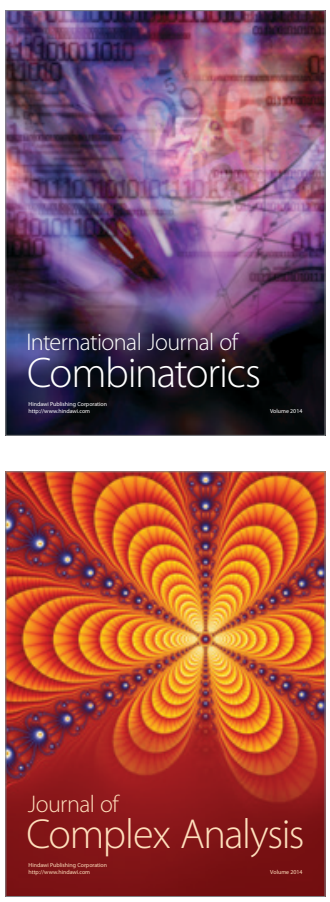

International Journal of

Mathematics and

Mathematical

Sciences
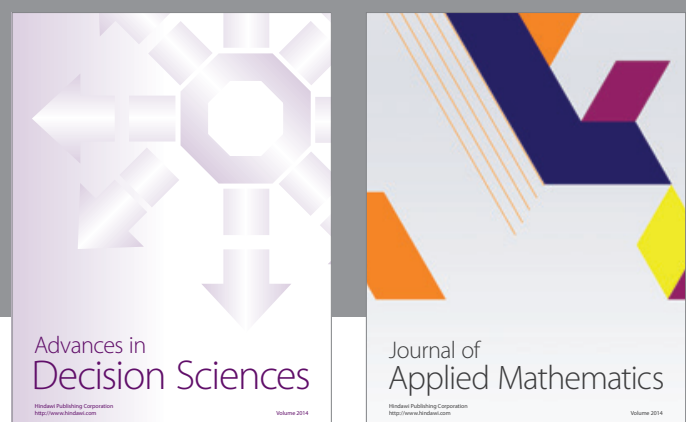

Journal of

Applied Mathematics
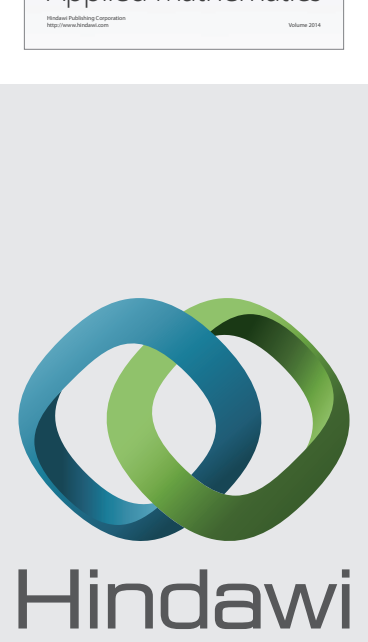

Submit your manuscripts at http://www.hindawi.com
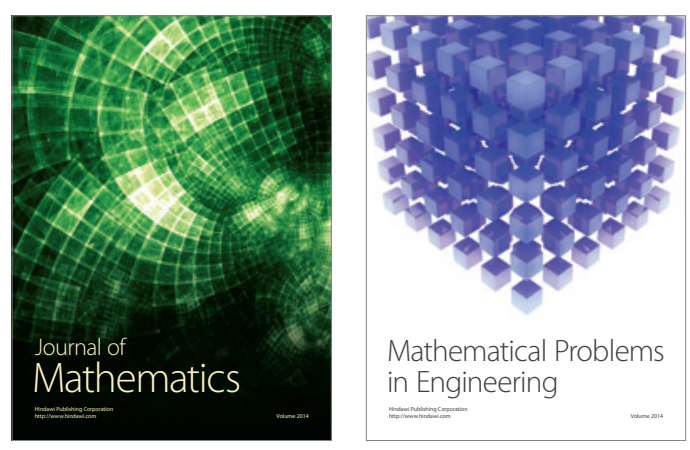

Mathematical Problems in Engineering
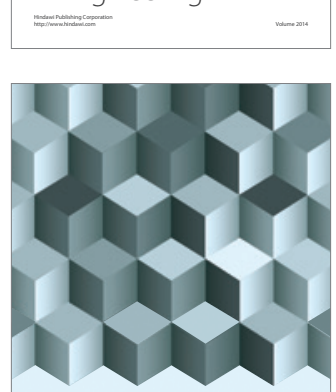

Journal of

Function Spaces
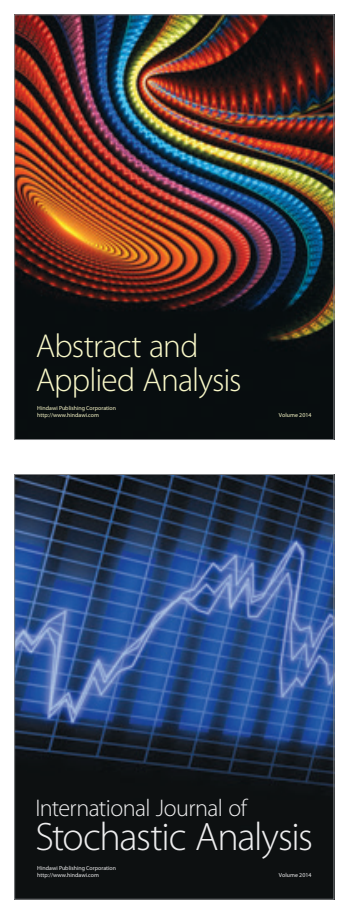

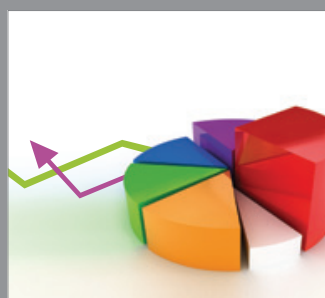

ournal of

Probability and Statistics

Promensencen
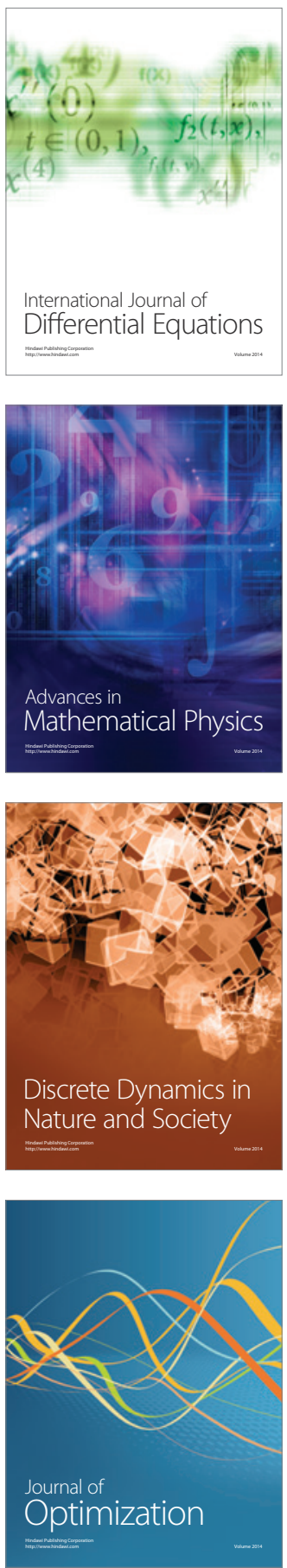